\title{
The Safety-Oriented Personality Style or Phobicentric Psychopathology: A Cross-National Replication Study
}

\author{
Patrick Bickersteth1, Samer J. Rudwan², Mahnaz Shojaee ${ }^{3}$, Tahereh Firoozi ${ }^{3}$ \\ ${ }^{1}$ PB Psychological Services, St Albert, Canada \\ ${ }^{2}$ University of Nizwa, Nizwa, Oman \\ ${ }^{3}$ University of Alberta, Edmonton, AB, Canada \\ Email: peaceofmindforum@gmail.com
}

How to cite this paper: Bickersteth, P., Rudwan, S. J., Shojaee, M., \& Firoozi, T. (2021). The Safety-Oriented Personality Style or Phobicentric Psychopathology: A Cross-National Replication Study. Psychology, 12, 1341-1363.

https://doi.org/10.4236/psych.2021.129084

Received: July 27, 2021

Accepted: September 10, 2021

Published: September 13, 2021

Copyright $\odot 2021$ by author(s) and Scientific Research Publishing Inc. This work is licensed under the Creative Commons Attribution International License (CC BY 4.0).

http://creativecommons.org/licenses/by/4.0/

(c) (i) Open Access

\begin{abstract}
Our replication study embarked on in the Middle East, used an Arabic translation of the original research tool, the Safety-Oriented Personality Style or Phobicentric Psychopathology Individual Questionnaire (SOPS/PCPIQ) that was employed in the North American study, which could identify adults with more serious chronic anxiety. SOPS/PCP, described as dimensional and neurobiologically-based is the hypothesized construct from which SOPS/PCPIQ is derived. SOPS/PCP, a brand-new formulation, arose serendipitously from clinical observations and followed in the new tradition of attempting to avoid characterizing personality disorder in the categorical and non-theoretical style of the previously influential Diagnostic Statistical Manual of Mental Disorders. The present study is intended to produce results that confirm that SOPS/PCP may be experienced outside North America. The results supported the hypotheses that the Arabic version of the SOPS/PCPIQ would demonstrate adequate reliability and validity. They further showed that individuals with a history of trauma exposure scored significantly higher than the other subjects. As such, the similar results from this Arabic study to those from Canada and the United States showed that this cross-national study successfully replicated the original North American findings. Discussion addresses the potential role of this study's psychopathology to provide meaningful contribution to the recognition and reduction of global anxiety disorder and help change the current direction of personality disorder research.
\end{abstract}

\section{Keywords}

NIMH-RDoC, Worldwide Burden of Anxiety, Dimensional Theory-Based, Fear-Anxiety Construct, Personality Disorder 


\section{Introduction}

The Safety-Oriented Personality style (SOPS) or Phobicentric Psychopathology (PCP) is a newly unveiled personality disorder, which has been empirically confirmed in two North American samples from Canada and the USA (Bickersteth et al., 2018a). That inaugural study presented SOPS/PCP as a previously unidentified type of anxiety disorder representing a viable diagnostic explanation of psychopathology. SOPS/PCP came to light following several years of observation and study of the behaviors and responses that certain individuals showed who were seen in the lead author's psychology clinic, which were also evident among some of his family members. In other words, people with differing family backgrounds, of various ages, raised in different parts of the world and who used different first languages, self-reported, were reported by their close relatives or were observed in the clinic to display the similar pattern of reactions, which eventually suggested that anxiety disorder was the cohering factor. The label SOPS/PCP was then applied to this constellation of responses to describe a disproportionate pattern of reacting to ordinary fear-anxiety situations and to broadly construe the construct in terms of a pervasive and persistent, reactive condition, which may be seen as triggered by actual or perceived threat. This condition is believed to develop through worsening stages, from adaptive fear in normal day-to-day emotional life to pathological reactivity, of which a very serious or severe expression of fear-anxiety would characterize SOPS/PCP as a disorder.

Because fear-anxiety is seen as the basis of this condition, an etiological and dimensional quality would empirically explain its progress from normal to dysfunctional functioning. Since the attributes of SOPS/PCP represent characteristics applicable to a normal emotional reaction of fear-anxiety, which, under specific circumstances develops into a worse form of a disordered pattern of persistent fearfulness or anxiety, and being emotion-based behavior, it is viewed as controlled by neurobiological activity. Accordingly, the fear-anxiety construct underlying SOPS/PCP is hypothesized to be dimensional and neurobiologically-based. As such, the SOPS/PCP neurobiological theory indicates the possibility to trace the range of an individual's expression of this personality from normal, adaptive fearfulness to an extreme that may be described as disturbed. In other words, in the long run these individuals would have internalized fear-anxiety as the major or persistent response pattern to most situations. As described by Bickersteth et al. (2018a): “... in an environment in which harm is perceived, expected or experienced, a person with this emotional condition will continually use disproportionate fear to interact with their social, physical and internal milieu." (p. 1635)

In prior times, the well-known Diagnostic and Statistical Manual of Mental Disorders (DSM) published by the American Psychiatric Association (APA, 2013), held sway as the North American leader in the diagnosis of psychological pathology. Over time however, the DSM's categorical system and non-theoretical framework provoked widespread criticism followed by calls for a new formula- 
tion of diagnostic criteria, founded on an etiological and dimensional basis. The disenchantment further led to attempts at developing a dimensional system (for example, Widiger \& Lowe, 2008; Trull, Tragesser, Solhan, \& Schwartz-Mette, 2007), within which to characterize and understand personality disorders, in contradistinction to the DSM's categorical formulation. The alternative framework, the Big 5 or Five Factor Model (FFM), which evolved, using a Domain-style presentation, was also not founded on etiology or theory.

The section on Mental, Behavioral or Neurodevelopmental Disorders (chapter 6) in the 11th Revision of the International Classification of Diseases and Related Health Problems (ICD-11) is the most recent European version of a compendium of diseases and health conditions, approved for global application by the World Health Organization (WHO). Commenting on it, Dan J. Stein and Geoffrey M. Reed (Stein, Szatmari, Gaebel et al., 2020) conclude that: “... although dimensional constructs have been included in a number of places in the ICD-11, this remains a largely categorical system that does not incorporate fine-tuned assessment of symptom variations" (p. 21).

In view of these issues, it is acknowledged as necessary to keep developing new theoretical models and taxonomic categories that are not included in international classifications (such as the DSM and ICD), which are worth further research, including further empirical and clinical investigations. The revealing of SOPS/PCP may be classified as one such effort. Notably, the process of laying out personality diagnostic principles has received guidance from the National Institute of Mental Health (NIMH) Research Domain Criteria (RDoC), which has stipulated, according to Cuthbert (2014), “... two stringent criteria: a) there had to be evidence for the dimension as a validated behavioral function, and b) there had to be evidence for a neural circuit or system that plays a preponderant role in implementing the function." (p. 29) The SOPS/PCP construct meets these criteria.

A clear significance of the SOPS/PCP research, beyond introducing a dimensional and neurologically based disorder, is in uncovering behaviors that previously have not been seen as related to the same group of anxiety disorder but in fact are. The link between these "disguised" reactions and typical anxiety reactions was confirmed in the study by Bickersteth et al. (2018a) which reported strong positive correlations between the test designed to study SOPS/PCP, namely SOPS/PCP Individual Questionnaire (SOPS/PCPIQ) and two well-established fear-anxiety tests (Beck Anxiety Inventory and Penn State Worry Questionnaire) and negative association with the Revised Anxiety Control Questionnaire (measuring the ability to regulate feelings of anxiety). In attempting to explain why these reactions were not previously recognized as arising from anxiety, Bickersteth and colleagues (2018a) state: “... these have 'morphed' over time into different chronic forms, due to shaping by internal and external emotion-based pressures, which in the case of SOPS/PCP are fear-directed." (p. 1653). Originally, SOPS/PCP was clinically described in detail, as: The Fear-Based Personality, in the lead author's book (Bickersteth, 2015, 
chapter 4).

Before SOPS/PCP identified certain behaviors as arising from anxiety disorder however, based on their common etiology, they appeared to be disparate behavioral descriptions. For example: apparent, insatiable craving for acknowledgement and praise; being prone to complaining and dissatisfaction; impatient, impulsive tendencies; easily offended, are all characteristics of SOPS/PCP, which would not immediately appear to be anxiety-based. As such, the true emotional identities of these attributes would not have been revealed and would have remained "hidden" and "scattered" in various, sometimes unrelated clusters and defined differently. For example, Body Dysmorphic Disorder (BDD), which may indicate fear of perceived physical abnormality that could attract negative evaluation (Fang \& Hofmann, 2010) could be classified under SOPS/PCP. Barclay conjectures that Borderline Personality Disorder (BLPD) originates from traumatic childhood experience (Barclay, 2015), which makes anxiety disorder a central contributor to BLPD. According to the research by Muris and colleagues “... shame plays a significant role in anxiety pathology” (Muris et al., 2018: p. 276), thus showing shame to be etiologically fear-based. This would allow for its definition to be: fear of discreditable exposure, which aligns it with an expression of SOPS/PCP. Since these disorders are not identified as fear-based, and currently belonging together in a common category, they are not recognized as such. Instead, these conditions have been assigned to different groupings. Based on the unique attributes associated with SOPS/PCP, then, and their verification in the North American context, it seems important to discover whether the result of a replication of the SOPS/PCP study would demonstrate this condition exists in other national/cultural settings, en route to empirically demonstrating its geographical universality and thus showing it to be a true, human predisposition. Previous contacts with psychologists outside Canada yielded the Middle East samples used in this replication study.

Since the main event hypothesized to cause SOPS/PCP is anxiety, due to some acquired or transmitted generic trauma experience, the SOPS/PCPIQ items were designed to select individuals who indicate trauma history (experience) apart from those who do not. Statistics are applied to differentiate these two groups. As such, the current study was embarked on, mainly to prove the hypothesis that a valid Arabic version of the SOPS/PCPIQ, tested on an Arabic sample, would yield results supporting the claim that SOPS/PCP exists outside Canada and the United States. In the light of an expected cross-national validation, we discuss the anticipated role of SOPS/PCP in the global scene, particularly, in relation to reducing the worldwide treatment gap and significantly easing the social burden of anxiety disorder. Personality disorder research methodology and cross national/cultural comparison issues are also addressed. Since the launching of SOPS/PCP represented the first occurrence of the study of this unique construct in the psychology literature, that initial research study (Bickersteth et al., 2018a), followed by a demographic analysis (Bickersteth et al., 2018b), together, consti- 
tute the only relevant existing literature.

\section{Materials and Methods}

\subsection{Sample}

The total number of registrations was 592 from two Arab countries: Oman and Syria. The psychological conditions of the study samples differ with respect to life conditions. The life conditions of Omani society are stable and the quality of life is of a high level. As would be expected, the life conditions of Syrian society, is unstable, due to the political situation that Syria has been going through for more than ten years. The participants from Syria are 272 and those from Oman are 320. All registrants participated in the study. The ages of Omani respondents ranged from 19 to 57 years ( $\bar{X}: 26.86$, s: 4.28). Syrian female respondents were 208 and the male respondents were 64, with ages ranging from 17 to 57 years ( $\bar{X}: 27.30$, s: 4.29). The data was collected via an online survey during the period between December 2019 and November 2020.

\subsection{Measure}

Arabic Version of SOPS/PCP Individual Questionnaire (SOPS/PCPIQ).

In order to demonstrate that SOPS/PCP was an actual personality disorder, the SOPS/PCPIQ (see Appendix 1) was developed and subjected to a number of studies for empirical verification (Bickersteth et al., 2018a). As reported in their study, the scale consisted of 29 items, which were answered according to a six-point Likert-style scale. The results demonstrated that the scale had suitable psychometric properties (internal reliability $=.59$ to .87 ).

The preparation of the Arabic Version of the SOPSIQ (see Appendix 2) utilized the following procedures:

- The English SOPS/PCPIQ text was translated into Arabic by the author and checked by the co-author, a fluent Arabic-English language specialist to ensure the accuracy and clarity of the translation.

- The necessary revisions were made and the resultant Arabic version was back translated to English. The result demonstrated an accurate English version.

- The Arabic version was then formatted for electronic application and the Internet link was disseminated on social media.

- The respondents were asked to participate voluntarily.

\subsection{Design}

This study's main goal was to produce a cross-national replication of the study of SOPS/PCP originally performed on a North American sample. To achieve this, the following steps were taken:

1) It needed to be confirmed that a valid Arabic version of the SOPS/PCPIQ, would yield similar results when tested on an Arabic sample. Therefore, to confirm the statistical qualities of the questionnaire, it was hypothesized that the Arabic, translated version of SOPSPCPIQ was reliable and valid. 
2) It was further hypothesized that a similar five factor structure as that obtained in the North American study would emerge from the Arabic study.

3) As with the North American study, in differentiating those who would be classified as exhibiting a dysfunctional level of SOPS/PCP, it would need to be shown that for the Arabic sample, the mean score obtained by the trauma-history subjects (represented by items 24 to 29) was significantly higher than that of the non-trauma-history subjects. This difference would be shown using t-tests. As such, the Cross-National, Empirical evidence of SOPS/PCP would be confirmed in the combined Omani-Syrian (Middle East) sample.

\section{Results}

\subsection{Descriptive Statistics}

Table 1 represents the normal distribution of the items. The range of mean score of the items are between .922 and 4.566. The range of standard deviation (SD) of the items is $.828<\mathrm{SD}<1.791$ which meets the criteria of normal distribution (Julious, 2005). More specifically, Item 26, "I have suffered from the effect of trauma or a difficult emotional life for at least two years", showed the highest SD, Item 29, "I and/or one parent has suffered a serious illness", showed the lowest mean score, and Item 15, “order, tidiness, and cleanness are very important", showed the highest mean score and the lowest SD. The normality of the responses was checked by examining the skewness and kurtosis tests too. As Table 1 showed Skewness is -.648 , which is in the normal range of acceptable values. Also, kurtosis is 2.402 , which is less than 3 and is also acceptable.

\subsection{Reliability}

The internal consistency of the scale was determined using Cronbach's alpha and mean inter-item correlation. As Table 2 shows the Cronbach's Alpha is a $=.814$ which indicates a relatively high $(\alpha>.70)$ internal consistency for the items.

\subsection{Exploratory Factor Analysis}

\subsubsection{Assumption Check}

Prior to conducting exploratory factor analysis (EFA), the properties of the measured variables and the sample size were checked. Considering the properties of the measured variables, we examined the linearity and multicollinearity of the items using the correlation matrix. The minimum item correlation is .003 and the maximum item correlation is .504. That said, the correlation between the items is linear and the items do not share lots of variance to violate the assumption of multicollinearity.

In order to check the properties of the sample size, we conducted KMO and Bartlett's test. Table 3 shows that the Chi-Square is significant, so we ensured that the sample size for conducting EFA is adequate. 
Table 1. Descriptive statistics.

\begin{tabular}{cccccccc}
\hline & Mean & Minimum & Maximum & Variance & N of Items & Skewness & Kurtosis \\
\hline Item Means & 2.792 & .922 & 4.567 & .776 & 29 & -.648 & 2.402 \\
Item Variances & 1.820 & .687 & 3.208 & .334 & 29 & & \\
\hline
\end{tabular}

Table 2. Reliability statistics.

\begin{tabular}{cc}
\hline Number of items & 29 \\
\hline Cronbach's Alpha & .814 \\
Cronbach's Alpha Based on Standardized Items & .816 \\
\hline
\end{tabular}

Table 3. KMO and bartlett's test.

\begin{tabular}{lcc}
\hline Kaiser-Meyer-Olkin Measure of Sampling Adequacy. & .865 \\
\hline \multirow{3}{*}{ Bartlett's Test of Sphericity } & Approx. Chi-Square & 4034.549 \\
& df & 406 \\
& Sig. & .000 \\
\hline
\end{tabular}

\subsubsection{Number of Factors}

The number of factor structure of the scale was determined using different tests including, scree test, parallel analysis, and model fit. The Scree Plot (Figure 1) represents the eigenvalues of the items. As Figure 1 shows, the sixth factor is on the dropping point of the plot. Given that the appropriate number of factors corresponds to the number of eigenvalues prior to the last major drop in the plot, five factors explain the majority of the variances in the scale.

The parallel analysis also represented the five-factor structure of the scale. Column 1 in Table 4 shows the O'Connor random data eigenvalues for 591 cases and 29 factors over 100 iterations. Comparing the random eigenvalues with the actual eigenvalues from the real dataset (Column 2, Table 4), we noticed that the eigenvalues of first five factors in the real dataset are higher than the random eigenvalues. However, the total eigenvalue in factor 6 is less than the random generated eigenvalue. Hence, the scree plot and parallel analysis suggested a five-factor structure for the scale.

A third technique which was used for determining the number of factors in the scale is examining the model fit. As Table 5 shows, the Chi-Square test for the model fit is significant which shows that the data fits the model.

\subsection{Factor Structure}

\subsubsection{Communality Estimates}

Table 6 represents the communality estimate as an indicator of the variances in each measured variable explained by the common factors in the model. The Initial column represents the squared multiple correlations based on the other measured variables. The Extraction column shows the variance in items explained by the extracted factors. The communalities of most of the items are 
moderate, except for items 1, 2, 3, 4, 6, 7, 13, 14, 18, 19, 20, 29 which show low $(<.3)$ communalities. Low communalities suggest that they are not loading on either of the 5 extracted factors, and, thus, it is comparatively unrelated to the other items in the scale.

\subsubsection{Factor Loading}

Table 7 shows the pattern matrix of the items. The loading values represent the impact of the factor on the item by controlling the effect of other factors on the item. The factor loading values can be greater than 1.00 or less than -1.00 (Nunnally \& Bernstein, 1994). As it can be seen from Table 7, 16 out of 29 items have clean factor loadings .3 or above on one factor and cross loadings less than .22 on other factors. Item $4,5,7,12,13,21$, and 23 are loaded on factor 1 ; no item has clear loading on factor 2; item 19 is loaded on factor 3; items 14, 15, 22 are loaded on factor 4; and items 25, 26, 27, 28, 29 are loaded on factor 5.

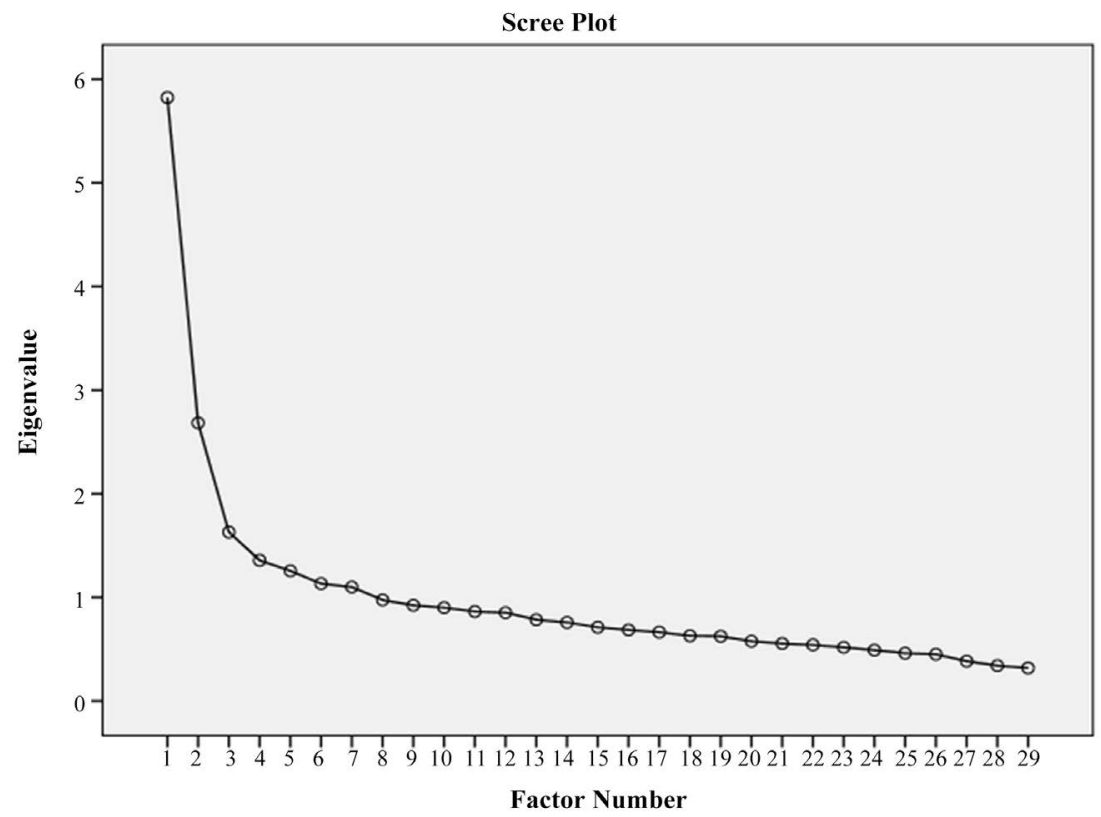

Figure 1. Scree plot.

Table 4. Parallel analysis.

\begin{tabular}{cccc}
\hline \multicolumn{2}{c}{ Random Data Eigenvalues } & \multicolumn{2}{c}{ Initial Eigenvalues } \\
\hline Roots & Percentile & Factors & Total \\
\hline 1.000000 & .54299 & 1 & 5.109 \\
2.000000 & .467090 & 2 & 2.142 \\
3.000000 & .422911 & 3 & .938 \\
4.000000 & .375613 & 4 & .662 \\
5.000000 & .336977 & 5 & .582 \\
6.000000 & .298743 & 6 & .533 \\
\hline
\end{tabular}


Table 5. Model fit.

\begin{tabular}{cccc}
\hline Chi-Square & $\mathrm{df}$ & RMSEA & Sig. \\
\hline 508.885 & 271 & .038 & .000 \\
\hline
\end{tabular}

Table 6. Communality estimates.

\begin{tabular}{|c|c|c|c|}
\hline \multicolumn{2}{|l|}{ Items } & \multicolumn{2}{|c|}{ Initial Extraction } \\
\hline 1 & It is normal to be unsure that you are safe from being harmed or hurt. & .125 & .063 \\
\hline 2 & It is easy for people to hurt others with unfair criticism or blame. & .151 & .122 \\
\hline 3 & I look for all the compliments and praise I am due & .148 & .084 \\
\hline 4 & $\begin{array}{l}\text { I often say so when it feels like life is unfair or things are not going my way } \\
\text { or in my favor. }\end{array}$ & .298 & .270 \\
\hline 5 & More than a few times a day I feel frustrated or upset. & .435 & .503 \\
\hline 6 & $\begin{array}{l}\text { It is very uncomfortable whenever others find fault with one's appearance } \\
\text { or image. }\end{array}$ & .188 & .161 \\
\hline 7 & It is difficult to wait for people or things. & .245 & .235 \\
\hline 8 & I probably speak or act in haste at times. & .295 & .300 \\
\hline 9 & I often regret things soon after I say or do them. & .313 & .325 \\
\hline 10 & $\begin{array}{l}\text { Some of my statements could be (or have been) described as sarcastic } \\
\text { humor. }\end{array}$ & .294 & .359 \\
\hline 11 & I may sometimes react very strongly or "lose it". & .372 & .434 \\
\hline 12 & $\begin{array}{l}\text { Many situations are difficult in a relationship (whether at work, socially, } \\
\text { in the family and/or in romantic situations). }\end{array}$ & .326 & .326 \\
\hline 13 & $\begin{array}{l}\text { The things that make a person very defensive or feel offended are always } \\
\text { around you. }\end{array}$ & .303 & .290 \\
\hline 14 & It is always very important to get as much information as there is. & .239 & .278 \\
\hline 15 & Order, tidiness and cleanliness are very important. & .245 & .355 \\
\hline 16 & $\begin{array}{l}\text { I have a definite liking or dislike for certain textures, colors, patterns } \\
\text { and/or motion. }\end{array}$ & .341 & .346 \\
\hline 17 & $\begin{array}{l}\text { It might seem to others that my behavior is opposite to or inconsistent } \\
\text { with other behaviors in similar situations. }\end{array}$ & .295 & .333 \\
\hline 18 & $\begin{array}{l}\text { Most of the time I take almost all statements and actions of others seriously } \\
\text { or "as is". }\end{array}$ & .082 & .035 \\
\hline 19 & Being on time and using time effectively are always very important. & .184 & .166 \\
\hline 20 & It is seldom necessary to disclose all of one's options. & .093 & .078 \\
\hline 21 & I worry often about many things. & .533 & .619 \\
\hline 22 & $\begin{array}{l}\text { "Do things my way" could well be my motto, because that always feels } \\
\text { better. }\end{array}$ & .237 & .309 \\
\hline 23 & Very often my mind is full of thoughts that come up again and again. & .401 & .404 \\
\hline 24 & $\begin{array}{l}\text { At least one of my parents (the person who raised me) may be described } \\
\text { as "a worrier". }\end{array}$ & .490 & .732 \\
\hline 25 & $\begin{array}{l}\text { At least one of my parents (the person who raised me) had emotional } \\
\text { problems when I was growing up. }\end{array}$ & .494 & .590 \\
\hline 26 & $\begin{array}{l}\text { I have suffered from the effects of trauma or a difficult emotional life for } \\
\text { at least two years. }\end{array}$ & .458 & .505 \\
\hline 27 & $\begin{array}{l}\text { I and/or one parent experienced a dangerous or very threatening personal, } \\
\text { political and/or military event/s for an extended period. }\end{array}$ & .411 & .533 \\
\hline 28 & I have been/am being bullied. & .414 & .479 \\
\hline 29 & I and/or one parent has suffered a serious illness. & .185 & .200 \\
\hline
\end{tabular}


Table 7. Pattern matrix.

\begin{tabular}{|c|c|c|c|c|c|c|}
\hline \multirow{2}{*}{ items } & & \multicolumn{5}{|l|}{ Factor } \\
\hline & & 1 & 2 & 3 & 4 & 5 \\
\hline 1 & $\begin{array}{l}\text { It is normal to be unsure that you are safe from being } \\
\text { harmed or hurt. }\end{array}$ & & & & .201 & -.116 \\
\hline 2 & $\begin{array}{l}\text { It is easy for people to hurt others with unfair criticism } \\
\text { or blame. }\end{array}$ & .134 & & -.100 & .215 & \\
\hline 3 & I look for all the compliments and praise I am due & .265 & & & & \\
\hline 4 & $\begin{array}{l}\text { I often say so when it feels like life is unfair or things } \\
\text { are not going my way or in my favor. }\end{array}$ & .542 & & & & \\
\hline 5 & More than a few times a day I feel frustrated or upset. & .642 & & -.110 & -.136 & .105 \\
\hline 6 & $\begin{array}{l}\text { It is very uncomfortable whenever others find fault } \\
\text { with one's appearance or image. }\end{array}$ & .224 & & -.112 & .163 & \\
\hline 7 & It is difficult to wait for people or things. & .306 & & -.120 & .140 & .129 \\
\hline 8 & I probably speak or act in haste at times. & & & -.531 & & \\
\hline 9 & I often regret things soon after I say or do them. & .178 & & -.476 & & \\
\hline 10 & $\begin{array}{l}\text { Some of my statements could be (or have been) } \\
\text { described as sarcastic humor. }\end{array}$ & & & -.611 & & \\
\hline 11 & I may sometimes react very strongly or "lose it". & .108 & & -.577 & & .102 \\
\hline 12 & $\begin{array}{l}\text { Many situations are difficult in a relationship (whether } \\
\text { at work, socially, in the family and/or in romantic } \\
\text { situations). }\end{array}$ & .349 & & -.156 & .127 & .173 \\
\hline 13 & $\begin{array}{l}\text { The things that make a person very defensive or feel } \\
\text { offended are always around you. }\end{array}$ & .364 & & -.131 & .136 & .108 \\
\hline 14 & $\begin{array}{l}\text { It is always very important to get as much information } \\
\text { as there is. }\end{array}$ & & .109 & .125 & .465 & \\
\hline 15 & Order, tidiness and cleanliness are very important. & & & & .587 & \\
\hline 16 & $\begin{array}{l}\text { I have a definite liking or dislike for certain textures, } \\
\text { colors, patterns and/or motion. }\end{array}$ & .178 & & -.388 & .178 & \\
\hline 17 & $\begin{array}{l}\text { It might seem to others that my behavior is opposite } \\
\text { to or inconsistent with other behaviors in similar } \\
\text { situations. }\end{array}$ & & & -.546 & & \\
\hline 18 & $\begin{array}{l}\text { Most of the time I take almost all statements and } \\
\text { actions of others seriously or "as is". }\end{array}$ & .122 & .130 & & & \\
\hline 19 & $\begin{array}{l}\text { Being on time and using time effectively are always very } \\
\text { important. }\end{array}$ & & .177 & .342 & & .141 \\
\hline 20 & It is seldom necessary to disclose all of one's options. & & & & .269 & \\
\hline 21 & I worry often about many things. & .714 & & & & \\
\hline 22 & $\begin{array}{l}\text { "Do things my way" could well be my motto, because } \\
\text { that always feels better. }\end{array}$ & & & & .541 & \\
\hline 23 & $\begin{array}{l}\text { Very often my mind is full of thoughts that come up } \\
\text { again and again. }\end{array}$ & .503 & & -.106 & .191 & \\
\hline 24 & $\begin{array}{l}\text { At least one of my parents (the person who raised me) } \\
\text { may be described as "a worrier". }\end{array}$ & .270 & -.801 & & & .105 \\
\hline 25 & $\begin{array}{l}\text { At least one of my parents (the person who raised me) } \\
\text { had emotional problems when I was growing up. }\end{array}$ & & -.607 & & & .324 \\
\hline 26 & $\begin{array}{l}\text { I have suffered from the effects of trauma or a difficult } \\
\text { emotional life for at least two years. }\end{array}$ & .202 & -.302 & & & .505 \\
\hline 27 & $\begin{array}{l}\text { I and/or one parent experienced a dangerous or very } \\
\text { threatening personal, political and/or military event/s } \\
\text { for an extended period. }\end{array}$ & -.107 & & -.130 & & .711 \\
\hline 28 & I have been/am being bullied. & & -.174 & & & .593 \\
\hline 29 & I and/or one parent has suffered a serious illness. & & & & & .444 \\
\hline
\end{tabular}




\subsubsection{Interfactor Correlation}

Table 8 represents the correlation among the factors. As it is clear from Table 8, factor 1, "Threat Sensitivity and Life Dissatisfaction" shows low correlation with factors $4(\alpha=.334)$, "Maladaptive Situational Control" and with factor 5 ( $\alpha$ $=.320$ ), "Traumatic Experience". Likewise, factor 2, "Harm Avoidance" has low correlation with factor 3 ( $\alpha=.328$ ), "Obsessive Tendency".

\subsection{The Effect of Trauma Items on the SOPS/PCPIQ Performance}

The effect of trauma items (item 24 to item 29) on other non-trauma items (items 1 to item 23) was examined by creating two groups of participants: with non-trauma history and trauma history. Participants with scores 0 or 1 were grouped as the non-trauma history group, and participants with scores 4 or 5 were classified as the trauma history group. Then, six independent t-tests were conducted to examine the effect of each of the trauma items on the performance of the two groups of participants on the SOPS/PSPIQ performance. It can be seen from Table 9 that when the participants were grouped based on Item 24, "at least one of my parents (the person who raised me) may be described as 'a worrier"', non-trauma group ( $\mathrm{M}=3.02, \mathrm{SD}=.62, \mathrm{~N}=124)$ performed lower $(\mathrm{t}=$ $-4.289 p<.001$, two-tailed $)$ than trauma group $(\mathrm{M}=3.39, \mathrm{SD}=.46, \mathrm{~N}=67)$. When the participants were grouped based on Item 25, "at least one of my parents (the person who raised me) had emotional problems when I was growing up", non-trauma group $(\mathrm{M}=3.08, \mathrm{SD}=.572, \mathrm{~N}=167)$ performed lower $(\mathrm{t}=$ $-2.870 p<.001$, two-tailed) than trauma group $(\mathrm{M}=3.342, \mathrm{SD}=.613, \mathrm{~N}=59)$. When the participants were grouped based on Item 26 , "I have suffered from the effects of trauma or a difficult emotional life for at least two years", non-trauma group $(\mathrm{M}=3.017, \mathrm{SD}=.598, \mathrm{~N}=139)$ performed lower $(\mathrm{t}=-5.021<.001$, two-tailed $)$ than trauma group $(\mathrm{M}=3.395, \mathrm{SD}=.470, \mathrm{~N}=88)$. When the participants were grouped based on Item 27 , "I and/or one parent experienced a dangerous or very threatening personal, political and/or military event/s for an extended period", non-trauma group $(\mathrm{M}=3.071, \mathrm{SD}=.570, \mathrm{~N}=186)$ performed lower $(\mathrm{t}=-3.748<.001$, two-tailed $)$ than trauma group $(\mathrm{M}=3.428, \mathrm{SD}=.459$, $\mathrm{N}=41)$. When the participants were grouped based on Item 28 , "I have been/am being bullied", non-trauma group $(\mathrm{M}=3.025, \mathrm{SD}=3.025, \mathrm{~N}=153)$ performed lower $(\mathrm{t}=-3.202<.001$, two-tailed $)$ than trauma group $(\mathrm{M}=3.320, \mathrm{SD}=.577$,

Table 8. Factor correlation matrix.

\begin{tabular}{cccccc}
\hline Factor & 1 & 2 & 3 & 4 & 5 \\
\hline 1 & 1.000 & .034 & -.404 & .334 & .320 \\
2 & .034 & 1.000 & .328 & .083 & -.249 \\
3 & -.404 & .328 & 1.000 & -.213 & -.203 \\
4 & .334 & .083 & -.213 & 1.000 & -.103 \\
5 & .320 & -.249 & -.203 & -.103 & 1.000 \\
\hline
\end{tabular}


Table 9. T-test results for the effects of trauma on SOPS/PCPIQ performance.

\begin{tabular}{|c|c|c|c|c|c|}
\hline Grouping at & Groups & $\mathrm{N}$ & Mean & $\mathrm{SD}$ & $\mathrm{T}(\mathrm{df})$ \\
\hline $\begin{array}{l}\text { At least one of my parents (the person who } \\
\text { raised }\end{array}$ & \multicolumn{2}{|c|}{ Non-trauma 124} & 3.022 & .623 & \multirow[t]{2}{*}{$-4.289(189)$} \\
\hline me) may be described as "a worrier" & Trauma & 67 & 3.395 & .464 & \\
\hline $\begin{array}{l}\text { At least one of my parents (the person who } \\
\text { raised }\end{array}$ & \multicolumn{2}{|c|}{ Non-trauma 167} & 3.089 & .572 & \multirow{2}{*}{$-2.870(224)$} \\
\hline $\begin{array}{l}\text { me) had emotional problems when I was } \\
\text { growing up }\end{array}$ & Trauma & 59 & 3.342 & .613 & \\
\hline \multirow{2}{*}{$\begin{array}{l}\text { I have suffered from the effects of trauma or a } \\
\text { difficult emotional life for at least two years }\end{array}$} & Non-trau & 139 & 3.017 & .598 & \multirow{2}{*}{$-5.021(225)$} \\
\hline & Trauma & 88 & 3.395 & .470 & \\
\hline \multirow{2}{*}{$\begin{array}{l}\text { I and/or one parent experienced a dangerous } \\
\text { or very threatening personal, political and/or } \\
\text { military event/s for an extended period }\end{array}$} & Non-trat & 186 & 3.071 & .570 & \multirow[b]{2}{*}{$-3.748(225)$} \\
\hline & Trauma & 41 & 3.428 & .459 & \\
\hline \multirow[b]{2}{*}{ I have been/am being bullied } & Non-trau & 153 & 3.025 & .584 & \multirow[b]{2}{*}{$-3.202(205)$} \\
\hline & Trauma & 54 & 3.320 & .577 & \\
\hline \multirow[b]{2}{*}{ I and/or one parent has suffered a serious illness } & Non-trau & 223 & 3.142 & .588 & \multirow[b]{2}{*}{$-1.048(245)$} \\
\hline & Trauma & 24 & 3.273 & .511 & \\
\hline
\end{tabular}

$\mathrm{N}=54$ ). When the participants were grouped based on Item 29, "I and/or one parent has suffered a serious illness", non-trauma group $(\mathrm{M}=3.142, \mathrm{SD}=.588$, $\mathrm{N}=223)$ performed lower $(\mathrm{t}=-1.048<.001$, two-tailed) than trauma group ( $\mathrm{M}$ $=3.273, \mathrm{SD}=.511, \mathrm{~N}=24)$.

Generally, results of the six independent $t$ tests revealed that participants with trauma-history showed significantly higher SOPS/PCPIQ mean score than participants with non-trauma history.

\section{Discussion}

\subsection{Findings of the Study: Successful Replication Gives SOPS/PCP Research Boost}

The main purpose of this study was to demonstrate that SOPS/PCP could be found outside North America, which this replication study should bring to light. The results confirm that this is the case. In providing such validation of this fear-anxiety construct, the results affirm that SOPS/PCP seems to offer a strong empirical platform to study anxiety disorder, at home and abroad. Ascribing it neurobiological and etiological framework allows for this condition to be tracked from a normal expression of fear-anxiety to disordered behavior. With a unique set of attributes, delineating “... a pattern of behavior that depicts a threat-sensitive and biologically promoted, disagreeable responsiveness to a variety of environmental, social, bodily and interpersonal cues ..." (Bickersteth, 2018a: p. 1632), SOPS/PCP is positioned to be influential in cross-national/cultural psychology theorizing. Barring any significant flaws being revealed that shows this condition to be illegitimate, future cross-national/cultural studies are likely to 
confirm its worldwide presence.

At this point however, it is understandable that researchers have tended to focus their investigative work in personality on mainly the behavioral patterns that the DSM categories and Big Five domains have presented, though both formulations lack etiological explanation and supporting neurobehavioral theory. Hopefully, with the guidance of the RDoC criteria, attention will begin to move to research paradigms supporting this newer trend of viewing personality as neurobiologically grounded, dimensional and empirically verifiable, now, presumably, started by the introduction of SOPS/PCP. Such transition is particularly crucial in studying the occurrence of anxiety disorder internationally, especially in areas where the study of personality is relatively nascent.

\subsection{Unique Characteristics of SOPS/PCP Promise to Help in Reducing Anxiety Treatment Gap}

In response to the current need for a reduction of the gap in treating anxiety disorders, it is reasonable to consider the role SOPS/PCP might play. Addressing the scope of the gap, a 31-member team of researchers (Alonso, et al., 2018) identified three groups of people, in a 21-country survey of people with a 12-month DSM-IV anxiety disorder (including PTSD). The groups respectively included those who, 1) perceived a need for treatment; 2) received any treatment; and 3) received possibly adequate treatment. The authors reported a large gap between groups 1) and 3), which led to their suggestion that there was a need for improving the recognition of anxiety disorders and for applying a higher quality of treatment.

The finding in our cross-national study provides an exact response to the need for detecting anxiety disorders, by identifying a new anxiety disorder. Because of its distinctive etiological origin in fear, treatment of SOPS/PCP along that trajectory would be logical and defensible. From that viewpoint, our study also contributes favorably to heeding the call for improved treatment quality.

On the other hand, cross-national/cultural research, which base studies of personality on the FFM, has demonstrated that its structural pattern may be found in a large number of countries and cultural settings. In psychology however, the ultimate goal is to improve mental health; and the FFM model does not appear to allow for a diagnostic evaluation to which treatment protocols may be attached. As such, the presence of the five factors across certain cultures, for example, as reported and defended by Terracciano and McCrae (2006), merely indicates that similar patterns of the FFM personality structure exist cross-nationally. As well, the FFM was developed from an apparently arbitrary selection of trait items and the domains (OCEAN) or traits comprising the Big 5 did not evolve as separate and independent units but as a unitary structure. So, while each pillar of the trait structure may be extrapolated for research comparison purposes it would seem untenable to attach values to these traits, as these do not define real-life observable behaviors, which may be counted. In other words, 
the cross-cultural findings of the FFM are about similar structural patterns of personality obtained from the cultural samples to which the FFM was applied, rather than about actual behaviors of individuals, which could be used to achieve healing in those cultures. Besides, as Bickersteth et al. (2018a) pointed out: "Essentially, the Big 5 model is the result of developing a list of lexically defined personality traits and subjecting it to factor analysis ..." (p. 1654) All said, the FFM or Big 5 model continues to remain, according to Matthews (1997), a “... structural description of various data sets" (p. 5). As such, the similarity in patterns would not be indicative of specific diagnostic behaviours of individuals, who could receive treatment or be counted epidemiologically. Even the use of factor analysis to confirm Big 5 traits in different cultures has been questioned. For example, Benet-Martinez (2007) states: "The problem is that one cultural group needs to be arbitrarily designated as the target factor structure, and, unfortunately, most studies set the imported structure (e.g., the English NEO structure obtained with U.S. participants) as the target toward which the other group structures will be shifted." (p. 173)

With respect to the treatment efficacy of DSM categories, their cross-cultural application is seen as a faulty endeavor partly because of a major shortcoming. According to Bredstrom (2019), “... much of the critique that was raised against the Cultural Formulation of DSM-IV is still relevant for today's version, i.e., that the manual is ethnocentric and rests upon a narrow understanding of culture." (p. 348) The DSM system, then, appears to be in default by virtue of a culturally exclusive guiding principle, which would limit the cross-cultural generality of its application, in the first place. Beyond this major shortcoming, cross-national/ cultural studies, which use the DSM diagnostic categories are always at risk of including samples with comorbid symptomatology (which some studies exemplify; for example, Inoue, et al., 2020). While such confounds can be avoided, for example, by implementing the recommendations of the American Academy of Child and Adolescent Psychiatry (Connolly et al., 2007), it may not be practical to apply this comprehensive protocol in a research study, especially in some cross-national/cultural settings. The American Academy's guidelines however, are themselves methodologically tainted, since they are based on the DSM process of identifying anxiety disorder, which has been widely shown to be flawed (for example, Thyer, 2015; Smith \& Combs, 2010).

The argument adduced so far shows both the FFM and DSM models to be insufficient and untrustworthy tools for the elucidation of cross-national/cultural anxiety disorder; but specifically, they are not valid or qualified as paradigms for evaluating anxiety personality disorder in a cross-cultural/national context. As such, both systems seem ill-equipped to help reduce the treatment gap in anxiety disorder, particularly cross-nationally or cross-culturally. This seems to leave SOPS/PCP in the singular position of being etiologically and neurobiologically equipped to make a meaningful contribution to identifying and reducing the global anxiety treatment gap. 


\subsection{Steps toward Reducing the Social Burden of Anxiety Disorder, Worldwide}

The globally pressing concerns that anxiety raises are the more serious because its chronic and severe state can be debilitating, as, for example, in the case of generalized anxiety disorder (GAD), particularly in view of its tendency to attract comorbid conditions. And, to compound the problem to some degree, it has been shown (Bickersteth et al., 2018a) and noted here, that the symptoms of SOPS/PCP may not be recognizable as a "typical" anxiety presentation in the way it is represented in the DSM diagnostic criteria. Therefore, as indicated by Pelletier et al. (2017), efficient probes are needed to reduce the social burden of anxiety disorder, through early detection and treatment. SOPS/PCP alone, being fully qualified at this point, may be the least problematic in answering that call, generally; but also, specifically, in the cross-national/cultural context. In fact, it is noteworthy that the SOPS/PCP construct ranks high in conforming with the NIMH's RDoC criteria. In the broader field of personality research, the RDoC framework guides investigations carried out nationally or cross-nationally towards providing “... understanding of how such factors as life events and the social environment interact with development to produce a range of observed outcomes" (Cuthbert, 2014: p. 30). To do so, researchers must follow a neurodevelopmental and dimensional path. SOPS/PCP's purported developmental history in a person and the empirical verification of the construct appear to exemplify this direction. As such, an implied route in applying the RDoC framework could be to start, as in the case of SOPS/PCP, with examining certain agreed upon emotion-based reactions. Such agreement would need to be based in the understanding that every action is in response to a certain emotion, even where that emotion is not initially obvious. The next stage would entail verifying that the selected behaviors and reactions, which appear to represent the emotion(s)-in-action are similar, if not identical, within an identified cohort of individuals; and then proceed to propose a paradigm (theory or theories) that best explains the behavioral pattern, without exception. This process circumvents the problem of co-morbid interference and strengthens the theory's validity. Arguably, from the vantage point of the "paradigm shift" indicated by SOPS/PCP, this research apparently provides concrete, rather than abstract, $\mathrm{RDoC}$ goal-achievement. It demonstrates the feasibility of identifying an emotion-based behavioral problem such as a fear-anxiety condition (construct) that cuts across DSM categories, falls under (using RDoC's language) "behavioral entities tied to neural systems" and may be subjected to "basic behavioral neuroscience" analysis (quotations from Whooley, 2014: p. 105).

Whooley's (2014) proffered rapprochement for defining, understanding and researching mental disorder is to seek “... a model that views the biological and social as always intertwined and interrelated and to figure out how they interact and combine to produce suffering." (p. 108) Indeed, it is necessary for brain patterns to interact with the external environment in order to manifest as, and 
be named, a behavior or personality disorder. To state Whooley's concept more comprehensively, as it applies to personality theory, and using SOPS/PCP's platform: Personality is a pattern of thinking, behaving and feeling that is the combined result of the biological and circumstantial (familial and environmental) imprint on a person's emotional and neurobehavioral development and expression.

That being the case, the results of our current replication study seem to confirm the cross-national reliability and adaptability of the SOPS/PCP construct to play a major role in determining the true weight of suffering caused by anxiety disorder around the world, as well as showing promise of being instrumental in its treatment and reduction.

\subsection{Addressing a Major Challenge in Personality Disorder Research around the World}

Delineating researchable entities inevitably rests on the definition of those chosen for study. How do the DSM and SOPS/PCP, being the only two diagnosticand therapy-oriented models, compare with respect to the procedural process of identifying symptoms, which are relevant to their respective formulation? In this regard, success is bound to be elusive, if not unachievable, for the DSM system cross-culturally, in view of fundamental diagnostic limitations. For example, the failure of DSM-5 to provide strong evidence of neurobiological underpinnings for its disorders likely occurred because of the absence of an etiological rationale, which would have guided the installation of a dimensional framework. And it is the same reason why, in the first place, imposing a dimensional model on a category-style foundation is unworkable. Without a defensible postulation of how a condition (defined by a set of symptoms) arises, one cannot determine its total progress through a continuum; nor by reference to their origin, definitively differentiate one symptom from another. If the etiological analysis of a reaction cannot be established in the Western context it will not be evident in a cross-cultural setting either. In all probability, the stumbling block is in not recognizing that the process of building an explanatory behavioural theory, on which symptom recognition depends, needs to go from observed behaviour to formulating a theory that explains it, which Bickersteth et al. (2018a) described as a behaviour-to-theory (BTT) approach, as opposed to proceeding from theory to behaviour (TTB). This is a pivotal diagnostic and therapeutic challenge for researchers in personality disorders. Consequently, an awakening from the comfortable familiarity of traditional TTB methodology to a brand-new world of BTT thinking needs to happen, to respond with trustworthiness to this urgent research problem. Accordingly, the SOPS/PCP construct is also founded on the BTT principle.

\subsection{Factors Pertinent to Cross National/Cultural Comparison of Anxiety Disorder Symptoms}

Problems relating equivalence, collectivistic/individualistic and emic-etic attributes 
introducing confounds in cross-national/cultural research, discussed in a large number of studies may turn out to be easier resolved when the underlying mechanism is known as it is with respect to SOPS/PCP. In such cases, the distinguishing symptoms caused by or indicative of a disorder are expected to be observed in every situation the disorder is investigated. For example, in any part of the world, a person experiencing a panic reaction would be expected to exhibit fear and palpitations, among other symptoms. Although different culturally-based reasons or attributions may be used to explain the occurrence of these reactions, only when the defined symptoms are present however, can it be said that the same condition is being measured in two or more cross-national/cultural locales. Ethnopsychology/ethnophysiology and contextual factors that differ from those in Western medicine but are present in a cultural setting with culture-specific meanings, indicated by Hofmann and Hinton (2014) are not descriptions of anxiety disorder. Rather, they are socially constructed attributions, the "things" believed to make people develop anxiety disorder. How these factors are construed is separate from the emotional content (e.g., fear, anger, shame etc.) of the condition in question. With respect to itiajin kyofusho (TKS) in Korean and Japanese cultures however, the description of the basis of this emotional problem is clear. In these instances, a commonly experienced emotion, fear, is the observable, basic characteristic of interest that defines the disorder, which can be measured and compared cross-nationally/culturally. Unfortunately, confirming that the emotion underlying TKS is, in fact, fear, when DSM categories are the source of the disorders being studied, complicates the picture because of the absence of their demonstrated biological (emotional) origin and probable co-morbid associations. When culturally based observations are about the emotional content, in this case, fear, as presented in behaviors such as TKS and SOPS/PCP, measurement and comparison are always equivalent cross-nationally/culturally. If the measurable entity, for example comprising an anxiety disorder, is not present it cannot be claimed that anxiety disorder in different contexts is the same or equivalent. Also, emic-etic considerations regarding cross-national/cultural comparison will not arise when the research construct is derived from a basic emotion, as in the case of anxiety personality disorder, and the aim is to investigate whether the same components are present cross-nationally/culturally. Since fear is the emotional basis of SOPS/PCP, comparing the results produced by it, obtained from cross-national investigations, is methodologically legitimate.

\section{Limitations and Future Research}

Because of the newness of SOPS/PCP in the cross national/cultural scene, there is no precedent, as was the case when first verified in North America, which now makes the North American (Canadian and USA) sample the only direct precedent. Therefore, despite empirical demonstration in two areas of the world, worldwide sample insufficiency at this stage may tend to limit the global gene- 
rality of SOPS/PCP. Large enough samples are needed worldwide, first of all, to further establish the universality of SOPS/PCP. Secondly, a larger sample pool will likely encourage the study of neuroscience-determined pathways and that of other biological mechanisms to fully understand SOPS/PCP. Because of its "cleaner" anxiety profile such investigations are likely to be aided by the greater ability of SOPS/PCP to more easily show the different stages of plasticity from earlier to the later "morphed" or chronic the cerebral pattern(s). The researchers further suggest conducting detailed studies that take into account the effects of political and social instability on this personality style. To the extent that "precision medicine" can be applied to psychological entities, that is to be the ultimate goal in diagnosing and treating SOPS/PCP.

\section{Conclusion}

The results of this cross-national study confirm that SOPS/PCP is the real-life phenomenon previously encountered within North America, and now in the Middle East. On the whole, as a fear-anxiety construct, SOPS/PCP exemplifies a research tool that's cross-nationally applicable and seems potentially adept at addressing the apparent behavior-theory problem (TTB vs. BTT) in personality research methodology. Being also capable of helping to accurately identify people with chronic anxiety problems, because of its etiology and measurability and, in tandem, promising to significantly contribute to the development of more effective treatment, SOPS/PCP therefore has potential to reduce the anxiety treatment gap and relieve the burden imposed by anxiety disorders, worldwide. Moreover, this fledging construct may yet come to be seen as comprising differentiated or distinguishable manifestations or spectrums of anxiety and personality disorder, which may come to light in a new and transparent classification system, prompted to some degree by this SOPS/PCP study.

\section{Ethics}

The Research Ethics Board of Concordia University Edmonton gave approval of the research. All participants were asked to participate voluntarily in this online survey.

\section{Conflicts of Interest}

The authors declare no conflicts of interest regarding the publication of this paper.

\section{References}

Alonso, J., Liu, Z., Evans-Lacko, S., Sadikova, E., Sampson, N., Chatterji, S., Abdulmalik, J., Aguilar-Gaxiola, S., Al-Hamzawi, A., Andrade, L. H., Bruffaerts, R., Cardoso, G., Cia, A., Florescu, S., de Girolamo, G., Gureje, O., Haro, J. M., He, Y., de Jonge, P. Thornicroft, G. et al. (2018). Treatment Gap for Anxiety Disorders Is Global: Results of the World Mental Health Surveys in 21 Countries. Depress Anxiety, 35, 195-208. https://doi.org/10.1002/da.22711 
APA American Psychiatric Association (2013). Diagnostic and Statistical Manual of Mental Disorders (5th ed.). https://doi.org/10.1176/appi.books.9780890425596

Barclay, M. (2015). A Deconstruction of Borderline Personality Disorder. Psychology, 6, 1187-1198. https://doi.org/10.4236/psych.2015.69117

Benet-Martinez, V. (2007). Cross-Cultural Personality Research: Conceptual and Methodological Issues. In R. W. Robins, R. C. Fraley, \& R. F. Krueger (Eds.), Handbook of Research Methods in Personality Psychology (p. 170-189). The Guilford Press.

Bickersteth, P. (2015). The Fear-Based Personality. In P. Bickersteth (Ed.), To Your Happiness: A Self-Healing Guide to Peace of Mind (Chapter 4). Amazon.

https://www.findinnerpeace.co/publications

Bickersteth, P., Zhang, X., \& Guo, Q. (2018a). Empirical Evidence for a New Class of Personality Disorder: The Safety-Oriented Personality Style or Phobicentric Psychopathology (SOPS/PCP) and Impact on Personality Psychology. Psychology, 9, 1630-1679. https://doi.org/10.4236/psych.2018.97098

Bickersteth, P., Zhang, X., \& Zhang, X. Z. (2018b). Gender and Age Differences in Safety-Oriented Personality Style or Phobicentric Psychopathology (SOPS/PCP). International Journal of Social Science Studies, 6, 22-31. https://doi.org/10.11114/ijsss.v6i12.3703

Bredstrom, A. (2019). Culture and Context in Mental Health Diagnosing: Scrutinizing the DSM-5 Revision. Journal of Medical Humanities, 40, 347-363. https://doi.org/10.1007/s10912-017-9501-1

Connolly, S. D., Bernstein, G. A., \& Work Group on Quality Issues (2007). Practice Parameter for the Assessment and Treatment of Children and Adolescents with Anxiety Disorders. Journal of the American Academy of Child and Adolescent Psychiatry, 46, 267-283. https://doi.org/10.1097/01.chi.0000246070.23695.06

Cuthbert, B. N. (2014). The RDoC Framework: Facilitating Transition from ICD/DSM to Dimensional Approaches That Integrate Neuroscience and Psychopathology. World Psychiatry, 13, 28-35. https://doi.org/10.1002/wps.20087

Fang, A., \& Hofmann, S. G. (2010). Relationship between Social Anxiety Disorder and Body Dysmorphic Disorder. Clinical Psychology Review, 30, 1040-1048. https://doi.org/10.1016/j.cpr.2010.08.001

Hofmann, S., \& Hinton, D. (2014). Cross-Cultural Aspects of Anxiety Disorders. Current Psychiatry Reports, 16, 450. https://doi.org/10.1007/s11920-014-0450-3

Inoue, T., Kimura, T., Inagaki, Y., \& Shirakawa, O. (2020). Prevalence of Comorbid Anxiety Disorders and Their Associated Factors in Patients with Bipolar Disorder or Major Depressive Disorder. Neuropsychiatric Disease and Treatment, 16, 1695-1704. https://doi.org/10.2147/NDT.S246294

Julious, S. A. (2005). Sample Size of 12 per Group Rule of Thumb for a Pilot Study. Pharmaceutical Statistics, 4, 287-291. https://doi.org/10.1002/pst.185

Matthews, G. (1997). An Introduction to the Cognitive Science of Personality and Emotion. In G. Matthews (Ed.), Cognitive Science Perspectives on Personality and Emotion (pp. 3-30). Elsevier. https://doi.org/10.1016/S0166-4115(97)80118-5

Muris, P., Meesters, C., \& Asseldonk, M. (2018). Shame on Me! Self-Conscious Emotions and Big Five Personality Traits and Their Relations to Anxiety Disorders Symptoms in Young, Non-Clinical Adolescents. Child Psychiatry and Human Development, 49, 268-278. https://doi.org/10.1007/s10578-017-0747-7

Nunnally, J. C., \& Bernstein, I. H. (1994). Psychometric Theory (3rd ed.). McGraw-Hill, Inc. 
Pelletier, L., O’Donnell, S., McRae, L., \& Grenier, J. (2017). The Burden of Generalized Anxiety Disorder in Canada. Health Promotion and Chronic Disease Prevention in Canada, 37, 54-62. https://doi.org/10.24095/hpcdp.37.2.04

Smith, G., \& Combs, J. (2010). Issues of Construct Validity in Psychological Diagnoses. In T. Millon, R. F. Krueger, \& E. Simonsen (Eds.), Contemporary Directions in Psychopathology: Scientific Foundations of the DSM-V and ICD-11 (pp. 205-222). Guilford Press.

Stein, D. J., Szatmari, P., Gaebel, W. et al. (2020). Mental, Behavioral and Neurodevelopmental Disorders in the ICD-11: An International Perspective on Key Changes and Controversies. BMC Medicine, 18, 21. https://doi.org/10.1186/s12916-020-1495-2

Terracciano, A., \& McCrae, R. (2006). Cross-Cultural Studies of Personality Traits and Their Relevance to Psychiatry. Epidemiology and Psychiatric Sciences, 15, 176-184. https://doi.org/10.1017/S1121189X00004425

Thyer, B. (2015). The DSM-5 Definition of Mental Disorder: Critique and Alternatives. In B. Probst (ed.), Critical Thinking in Clinical Assessment and Diagnosis. Essential Clinical Social Work Series (pp. 45-68). Springer. https://doi.org/10.1007/978-3-319-17774-8 3

Trull, T., Tragesser, S., Solhan, M., \& Schwartz-Mette, R. (2007). Dimensional Models of Personality Disorder: Diagnostic and Statistical Manual of Mental Disorders Fifth Edition and Beyond. Current Opinion in Psychiatry, 20, 52-56.

https://doi.org/10.1097/YCO.0b013e328010c838

Whooley, O. (2014). Nosological Reflections: The Failure of DSM-5, the Emergence of RDoC, and the Decontextualization of Mental Distress. Society and Mental Health, 4, 92-110. https://doi.org/10.1177/2156869313519114

Widiger, T. A., \& Lowe, J. R. (2008). A Dimensional Model of Personality Disorder: Proposal for DSM-V. Psychiatric Clinics of North America, 31, 363-378.

https://doi.org/10.1016/j.psc.2008.03.008 


\section{Appendix 1}

The SOPS/PCP Individual Questionnaire (SOPS/PCPIQ)

Select your response choice to express how you feel or think about each statement

$5=$ Very Often

$4=$ Often

$3=$ Occasionally

2 = Rarely

$1=$ Very rarely

$0=$ Never

\section{ITEM RESPONSE}

1. It is normal to be unsure that you are safe from being harmed or hurt.

0

1

2. It is easy for people to hurt others with unfair criticism or blame.

$\begin{array}{llllll}0 & 1 & 2 & 3 & 4 & 5 \\ \text { Never } & & & & \end{array}$

Always

Always

3. I look for all the compliments and praise I am due

0

Never

Always

I often say so when it feels like life is unfair or things are not going my

4. way or in my favor

Never

Always

0

5. More than a few times a day I feel frustrated or upset

Never

0

It is very uncomfortable whenever others find fault with one's

appearance or image

Never

7. It is difficult to wait for people or things

0

Never

0

8. I probably speak or act in haste at times.

Never

9. I often regret things soon after I say or do them.

0

Never

Some of my statements could be (or have been) described as sarcastic 0

10. humor.

Never

11. I may sometimes react very strongly or "lose it"

0

Never

Many situations are difficult in a relationship (whether at work, socially, in the family and/or in romantic situations)

0

Never

The things that make a person very defensive or feel offended are

always around you

0
Never

0

14. It is always very important to get as much information as there is.

Never

15. Order, tidiness and cleanliness are very important

0

Never

I have a definite liking or dislike for certain textures, colors, patterns and/or motion

0
Never

It might seem to others that my behavior is opposite to or inconsistent 0

with other behaviors in similar situations

Never

5

Always

5

Always

5

Always

5

Always

5

Always

5

Always

5

Always

5

Always

5

Always

5

Always

5

Always

5

Always

5

Always

5 Always 


\section{Continued}

\begin{tabular}{|c|c|c|c|c|c|c|c|}
\hline 18. & $\begin{array}{l}\text { Most of the time I take almost all statements and actions of others } \\
\text { seriously or "as is" }\end{array}$ & $\begin{array}{l}0 \\
\text { Never }\end{array}$ & 1 & 2 & 3 & 4 & $\begin{array}{l}5 \\
\text { Always }\end{array}$ \\
\hline 19. & Being on time and using time effectively are always very important & $\begin{array}{l}0 \\
\text { Never }\end{array}$ & 1 & 2 & 3 & 4 & $\begin{array}{l}5 \\
\text { Always }\end{array}$ \\
\hline 20. & It is seldom necessary to disclose all of one's options & $\begin{array}{l}0 \\
\text { Never }\end{array}$ & 1 & 2 & 3 & 4 & $\begin{array}{l}5 \\
\text { Always }\end{array}$ \\
\hline 21. & I worry often about many things. & $\begin{array}{l}0 \\
\text { Never }\end{array}$ & 1 & 2 & 3 & 4 & $\begin{array}{l}5 \\
\text { Always }\end{array}$ \\
\hline 22. & $\begin{array}{l}\text { "Do things my way" could well be my motto, because that always feels } \\
\text { better }\end{array}$ & $\begin{array}{l}0 \\
\text { Never }\end{array}$ & 1 & 2 & 3 & 4 & $\begin{array}{l}5 \\
\text { Always }\end{array}$ \\
\hline 23. & Very often my mind is full of thoughts that come up again and again. & $\begin{array}{l}0 \\
\text { Never }\end{array}$ & 1 & 2 & 3 & 4 & $\begin{array}{l}5 \\
\text { Always }\end{array}$ \\
\hline 24. & $\begin{array}{l}\text { At least one of my parents (the person who raised me) may be } \\
\text { described as "a worrier" }\end{array}$ & $\begin{array}{l}0 \\
\text { Never }\end{array}$ & 1 & 2 & 3 & 4 & $\begin{array}{l}5 \\
\text { Always }\end{array}$ \\
\hline 25. & $\begin{array}{l}\text { At least one of my parents (the person who raised me) had emotional } \\
\text { problems when I was growing up }\end{array}$ & $\begin{array}{l}0 \\
\text { Never }\end{array}$ & 1 & 2 & 3 & 4 & $\begin{array}{l}5 \\
\text { Always }\end{array}$ \\
\hline 26. & $\begin{array}{l}\text { I have suffered from the effects of trauma or a difficult emotional life } \\
\text { for at least two years }\end{array}$ & $\begin{array}{l}0 \\
\text { Never }\end{array}$ & 1 & 2 & 3 & 4 & $\begin{array}{l}5 \\
\text { Always }\end{array}$ \\
\hline 27. & $\begin{array}{l}\text { I and/or one parent experienced a dangerous or very threatening } \\
\text { personal, political and/or military event/s for an extended period }\end{array}$ & $\begin{array}{l}0 \\
\text { Never }\end{array}$ & 1 & 2 & 3 & 4 & $\begin{array}{l}5 \\
\text { Always }\end{array}$ \\
\hline 28. & I have been/am being bullied & $\begin{array}{l}0 \\
\text { Never }\end{array}$ & 1 & 2 & 3 & 4 & $\begin{array}{l}5 \\
\text { Always }\end{array}$ \\
\hline 29. & I and/or one parent has suffered a serious illness & $\begin{array}{l}0 \\
\text { Never }\end{array}$ & 1 & 2 & 3 & 4 & $\begin{array}{l}5 \\
\text { Always }\end{array}$ \\
\hline
\end{tabular}

NB: The range of 4 - 5 on the Likert scale and clinical judgement may be used to establish pathological level, though further studies incorporating neuroscience and other findings may contribute to establishing a "cut-off" point. 


\section{Appendix 2}

The SOPS/PCP Individual Questionnaire (SOPS/PCPIQ), Arabic Version

اقرأ من فضلك كل عبارة بعناية، وقرر إلى أي حد تصف كل منها مشاعرك وسلوكك وآرائك، ثم بين مدى انطباقها عليك بوجه

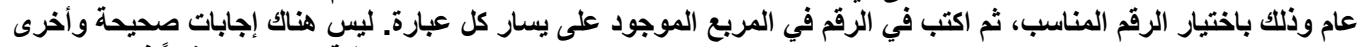
خاطئة، ولا تفكر كثيراً في كل عبارة.

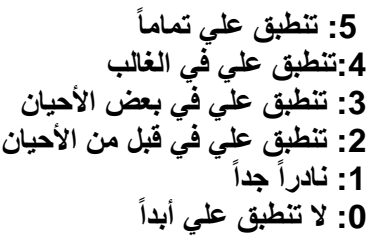

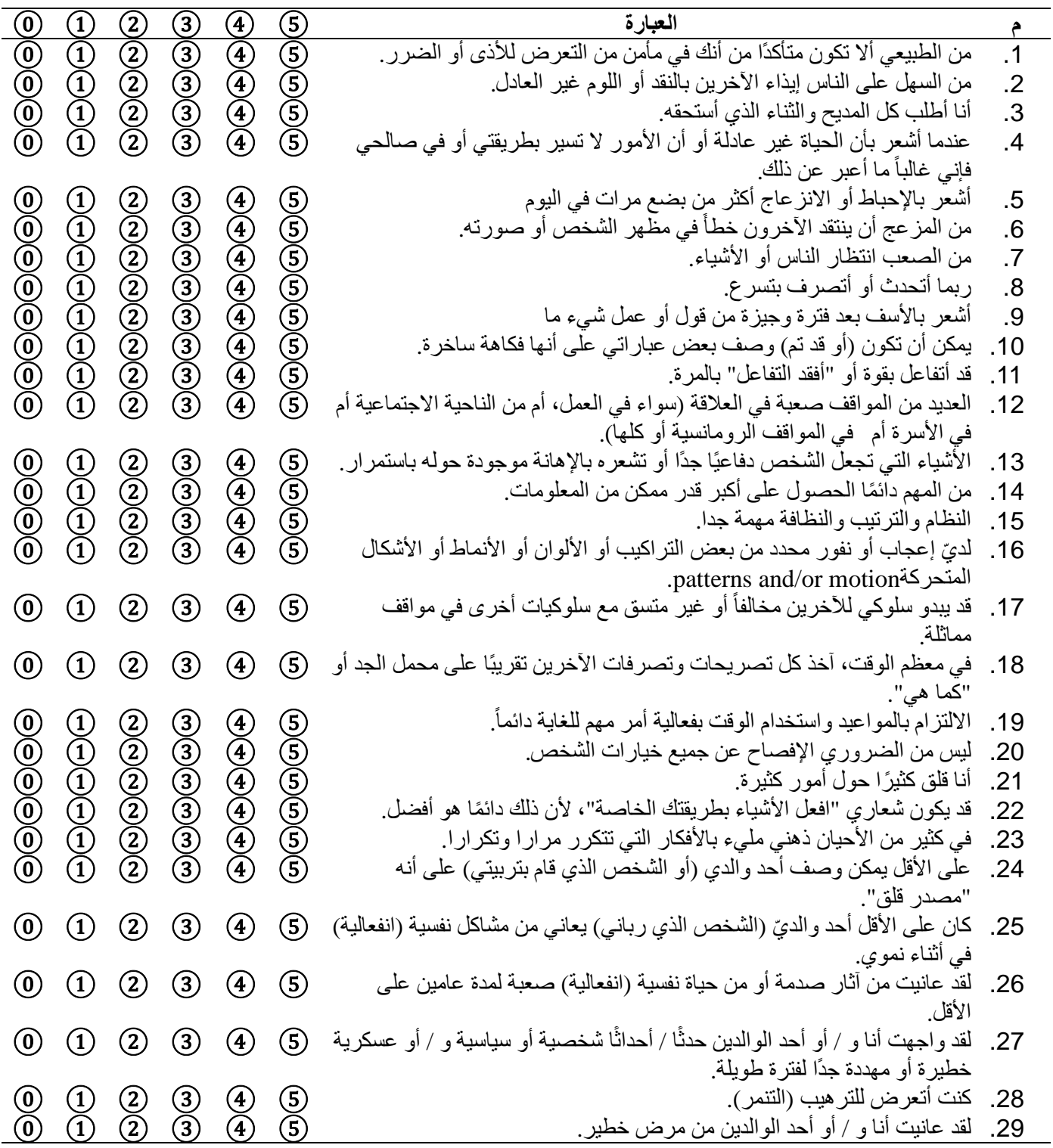

